\title{
Towards trust and trust building in a selected Cloud gaming virtual community
}

\author{
Yulin Yao ${ }^{1}$ Victor Chang ${ }^{2,3}$ \\ 1. Freelance Consultant, Anastaya, United Kingdom, UK \\ 2. School of Computing, Creative Technologies and Engineering, \\ Leeds Metropolitan University, Leeds, UK. \\ 3. Electronics and Computer Science, University of Southampton, Southampton, UK \\ yulinyao.forever@gmail.com \\ V.I.Chang@leedsmet.ac.uk
}

\begin{abstract}
Trust is an increasingly important factor in the virtual communities (VCs), since trust between different members can determine the long-term collaboration. Our approach is to examine on trust building on a Cloud gaming VC. We present the related literature and set four major hypotheses based on our literature review. Survey questions were designed based on our hypotheses and sent out to Chinese participants. We collected 100 valid sample size and presented the data demographics. We analyze our data which has p-values 0.05 and below for all categories of our four hypotheses. We use one-way ANOVA to compare the current and previous data and explain the interpretation of our analysis. Results show that there is a high extent of accuracy, consistency and support our four hypotheses. To support the case that online gaming is an aspect of emerging Cloud, we show examples about role-playing game, mission in the game, transferring trust to friendship and other games in demands. We discuss our limitation of this research and explain our future directions to improve on these aspects.
\end{abstract}

\section{Introduction}

The development of both the Internet and Web 2.0 have changed the way that organizations operate, which include the rise of e-commerce and online gaming platforms. The profits generated by the online gaming alone have more than US \$ 2.1 billions (Lehdonvirta, 2009). Market research conducted by the iResearch Consulting Group, a marketing research Chinese firm, stated that $45.3 \%$ of China's virtual communities (VCs) emerged in 2007 (iRsearch, 2007 a). The role of VCs is not only a platform for gaming but social interactions, since web users express themselves, interact with each other, find partners for missions, form clans to protect themselves against enemies and eventually establish their social networks. These VCs can develop into ecommerce sites with a Consumer-to-Consumer (C2C) model. According to iResearch (2007 b), these VCs can be known as e-Commerce oriented Social Networks (ESNs), which involves transactions by VC members and has been developed into an emerging business model in China. ESNs are considered promising by practitioners and researchers for reasons as follows. First, VCs appeal to a large number of online users due to their interactivity, openness and the ability to connect many others with similar interests. In countries like China, not all the voices can be heard, VCs provide platforms for online 
users to share and discuss games of interest. There is an estimate of 500 million VC users by the end of 2013 (Fotis, Buhalis and Rossides, 2011). Second, VCs help companies execute their targeted marketing campaigns since VC forums attract people with similar interests and passion. Third, Bughin and Zeisser (2001) and iResearch (2007 a; 2012) assert that there is a higher customer conversation rate than other similar businesses such as portals, information service providers and content providers. Additionally, activities organized for VC members can help improve the customer loyalty.

There are companies that provide existing VC customers the platform for transactions. These include buying or selling products or services, or improvement of existing transaction services for VC customers. iRsearch (2008) assert that C2C type of ecommerce has accounted to $93 \%$ of all the internet transactions in 2008. Additionally, there are four active $\mathrm{C} 2 \mathrm{C}$ platforms in China that works for gaming communities, including Taobao (part of Alibaba), TOM and eBay, PaiPai (provided by Tencent) and Baidu. The percentage of their market dominance and details of each service provider is presented in technical reports by iResearch (2007 a; 2007 b; 2012). All the major VCs in China offer links and services to these four major service providers, who rely heavily on VC members to communicate and share the knowledge between members. The operational team of the VCs and the dynamic interactions between members also encourage members themselves to take part in C2C transactions (iResearch 2007 a; 2012 a; Lu and Zhou, 2007). Lu and Zhou (2007) state that no previous work has empirically tested the relationship between $\mathrm{VCs}$ and $\mathrm{C} 2 \mathrm{C}$ e-commerce, and thus finding out factors contributing to the successful delivery between $\mathrm{VCs}$ and $\mathrm{C} 2 \mathrm{C}$ e-commerce.

$\mathrm{C} 2 \mathrm{C}$ platforms require members to communicate effectively in VCs and convince VC members to become $\mathrm{C} 2 \mathrm{C}$ buyers and sellers to stay competitive and economically sustainable. However, trust is a challenge that hinders $\mathrm{C} 2 \mathrm{C}$ development in China since more than fifty percent of offline customers do not purchase online due to their distrust of the digital platforms (Analysys, 2008). Some concerns are as follows. First, a large number of counterfeit products were sold on these websites, although efforts of counterfeit product prevention by some C2C websites were made (Fitzpatrick, 2006; Wang, 2009). Second, some C2C sellers place product pictures from magazines of high quality products but post inferior products. Based on a survey conducted by Sina.com (2009), more than 70\% of users had experienced of buying counterfeit or inferior products. Some feedback suggested there was no point in reporting the fraud to police, since laws were not established until 2009.

In our previous experience of managing VCs and running a consulting firm, VCs can offer an effective way of overcoming trust building for two reasons. First, VCs allow members to obtain the information they look for, and also support from each other. The majority of members can be influenced by VC leaders and members with specialized knowledge or experience in their areas. This is particularly true that "word-of-mouth" marketing can be effective in the C2C e-commerce (iResearch 2007 a; 2012). Second, $\mathrm{VCs}$ can be used a medium to facilitate trust building in $\mathrm{C} 2 \mathrm{C}$ e-commerce. To support this point, $\mathrm{Ba}$ (2001) used game theory to illustrate the effectiveness of a community agent, which can be more effective than an individual agent to provide the continuity of 
trust building processes. The reason is that a community with a long lifespan could overcome the limitation of an individual agent which might produce fraud or cheat other clients during the last transactions. Companies could go into "bust" without warning that leaders went away with millions and only a minority was caught. Ba (2001) also showed that it costs less to build trust at the community-level than at the individual level.

To take the trust issue forward, we examine trust building in VCs on gaming which have $\mathrm{C} 2 \mathrm{C}$ e-commerce services. We also study how our chosen gaming $\mathrm{VC}$ affects consumers to obtain information and purchase from $\mathrm{VC}$ websites. We focus on identifying and quantifying the relationship between the $\mathrm{VCs}$ and $\mathrm{C} 2 \mathrm{C}$ e-commerce services and examining the conversion from VC members to $\mathrm{C} 2 \mathrm{C}$ buyers. To address relevant issues and theme for Cloud Computing, we use a Cloud gaming virtual community as a case study. We set our hypothesis, send out surveys, analyze data and explain our findings. The paper is organized as follows. Section 2 provides background information about the chosen Cloud gaming. Section 3 presents the related literature for virtual communities and trust. Section 4 explains the four major hypotheses set for this research. Section 5 describes the research methodology, the types of survey questions for four major hypotheses, data collection and data analysis. Section 6 presents discussion topics including data interpretation and limitation of this research. Section 7 describes outlines future direction of this research. Section 8 gives sums up this paper and outlines the future work.

\section{Background information about the Cloud Gaming}

As discussed in Section 1, online gaming has a huge market potential and development with rapid increases in revenues and user community year by year. To support millions of users with the real-time support, Cloud Computing has become a popular option to offer multi-tenancy and dynamic scaling of servers with virtual machines. When a virtual machine is in demand, the Cloud can check which server is free of virtual machine and dynamically assign the virtual machines to the players, so that when the resource can be flexibly assigned to ensure that the rate of utilization can be high. This approach is called the resource allocation model which identifies which part of the Cloud needs more demands, and then assigns players to places with less demands.

Although the increase in membership numbers is seen in the online community, trust is a main concern among some participants. Our study can illustrate the significance in this area. While trust is a factor which can be different based on the communities, the culture and the mission of the communities, our work presents the case that online gaming is an aspect of emerging Cloud. In this section, we aim to illustrate why online gaming can attract much attention and has created a platform to develop friendship and business opportunities. Instead of surveying another group of 100 participants with probable different outcomes, we use a walkthrough approach to explain why online gaming has the potential and the market for Cloud Computing. 


\subsection{Relevance of Cloud gaming}

There is an increased number of games hosted in the Cloud. The benefits include the consolidation of resources and cost-savings for service providers and the ease of use and accessibility for players (Chang et al., 2012; Chang et al, 2013). These games include Warcraft, which allow the players to choose which virtual servers they can play, and they can also change to different virtual servers for different types of purposes. For example, one virtual server is used as a server for having a mission in progress and other virtual server can be used to train the player's skill and experience to higher levels. The games developed by the East have adopted similar approaches, which include role-play games (RPG) hosted in the service providers' Cloud. The advantages of doing so allow players from different types of network and computing resources to play with each other. Resource allocation and monitoring can be consolidated by the service providers so that the players may get the better services in terms of having less waiting time in queues and less time reaching their destination if the particular virtual servers are in big demands. In this research, we focus on the study of trust in the Cloud gaming community since it is a fast-paced area in Cloud Computing and there are not many studies investigating the trust issue in the Cloud VCs.

\subsection{A selected Cloud Gaming virtual community}

The gaming VC has a website at http://jx3.xoyo.com/ which offers RPG Cloud gaming. Their aim is to foster individual role in one of the ten major clans. The purpose is to train your main character with the improved level of fighting skills, experiences and equipment. Each ten clans have four characters: adult male, adult female, teenage male and teenage female. All the players should aim for completing missions and kill the 'boss' at every major stage of the game. All the players, even they are strangers, can work together to complete their missions. Advanced equipment can be obtained through killing the big boss at each stage, or purchased by the virtual coins in the game, or real money amongst players. For example, if player A planed to obtain advanced equipment for his character, he was a beginner and would like to get them through payment of real money. Player B is a veteran player and has this advanced equipment and offers it for US $\$ 1$. Both players chatted in the bulletin board system (BBS), or in private conversations in the chat room of the game for price negotiation. The price might come down to US 80 cents, or the seller could offer multiple items for another price such as US \$5, and player A would decide. Once they agreed to the deal, player A then paid through a trusted thirdparty vendor such as Taobao (2007). This completed the C2C payment. See Figure 1. 


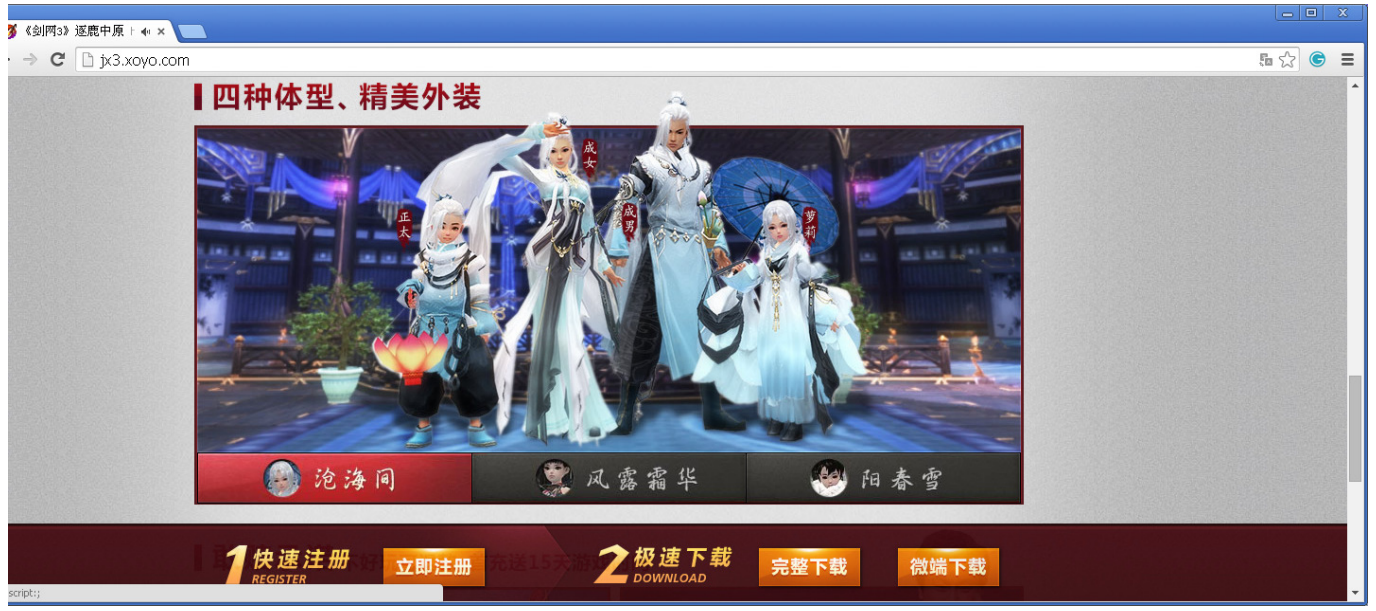

Figure 1: The Cloud gaming virtual community (VC) on http://jx3.xoyo.com/

\subsection{Role playing game}

The selected Cloud gaming virual community (VC) in this paper is a typical roleplaying game (RPG) that allows each member to select a number of different characters, but a main character can only be used each time. For example, if player A has characters of a sword fighter of the clan A, a witch, and a martial artists (without weapons) of Clan $\mathrm{B}$, he or she can only use one of his $\mathrm{VC}$ role each time. When player $\mathrm{A}$ is in the game, he or she needs to find out his mission, the equipment required for a successful mission and all types of requirements (such as collection of expected items, or alliance with another Clan, or killing the big boss of each stage).

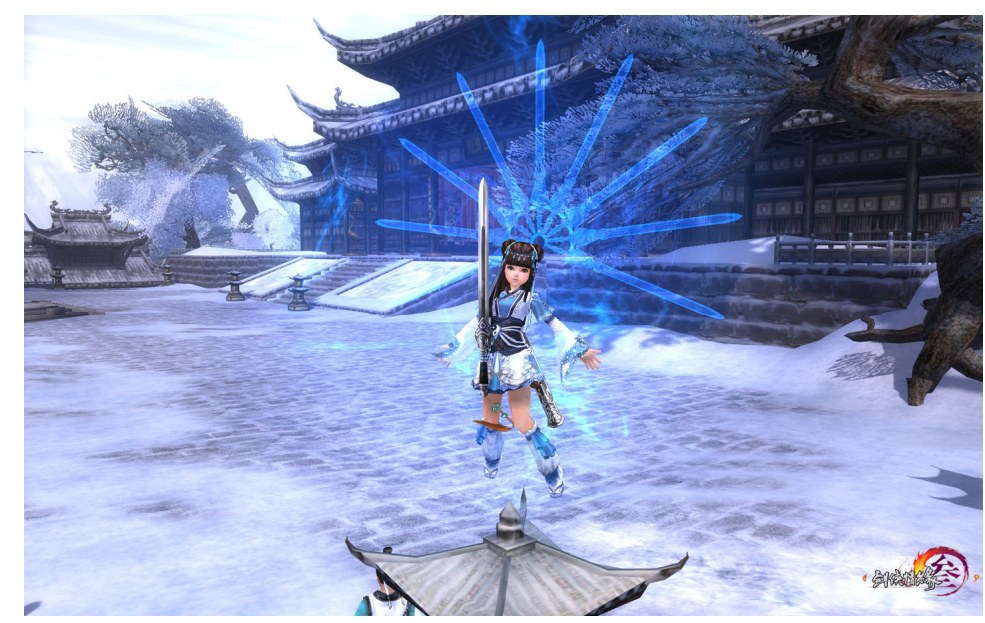

Figure 2: An example showing a female teenage fighter improving her skills

Player A should also learn about how to trade with characters designed by the game and other players who were members in the VC. There are other dialogue boxes in the game allow player A to interact with other players. Player A can also learn special techniques to improve his or her martial arts, which are crucial while fighting off enemies, including the bosses designed for the game, or missions involved with fighting in teams. Figure 2 shows an example that player A has chosen a female teenage fighter 
rehearing her sword fighting skills. She can power up within one second to demonstrate her special killing techniques.

\subsection{Missions in the game}

As discussed in section 5.1, there are different types of missions to be completed. This can be small tasks such as delivering goods to a particular destination that requires the player to fight off all types of enemies in the process of making deliveries. It can be huge tasks such as fighting and killing off the boss of each stage, which is equivalent of destroying an entire Clan that includes hundreds of highly-skilled "demon fighters". This is why the online purchase and communications (presented in Sections 5.1 and 5.2) play an important role in making trust into actions. While players purchase the tools for the automated playing to enhance their fighting skills and the tools for the enhanced life to make them less vulnerable to death, players have better chances of survival and getting the mission completed. Figure 3 shows a mission in progress which involves fighting off enemies and dialogues with team mates. The player needs to get to a particular destination to confirm that the mission is completed, which is shown in Figure 4. The benefit of getting missions completed will get the bonus points up, which can be converted to the improvement in the player's skills, fame and experience.

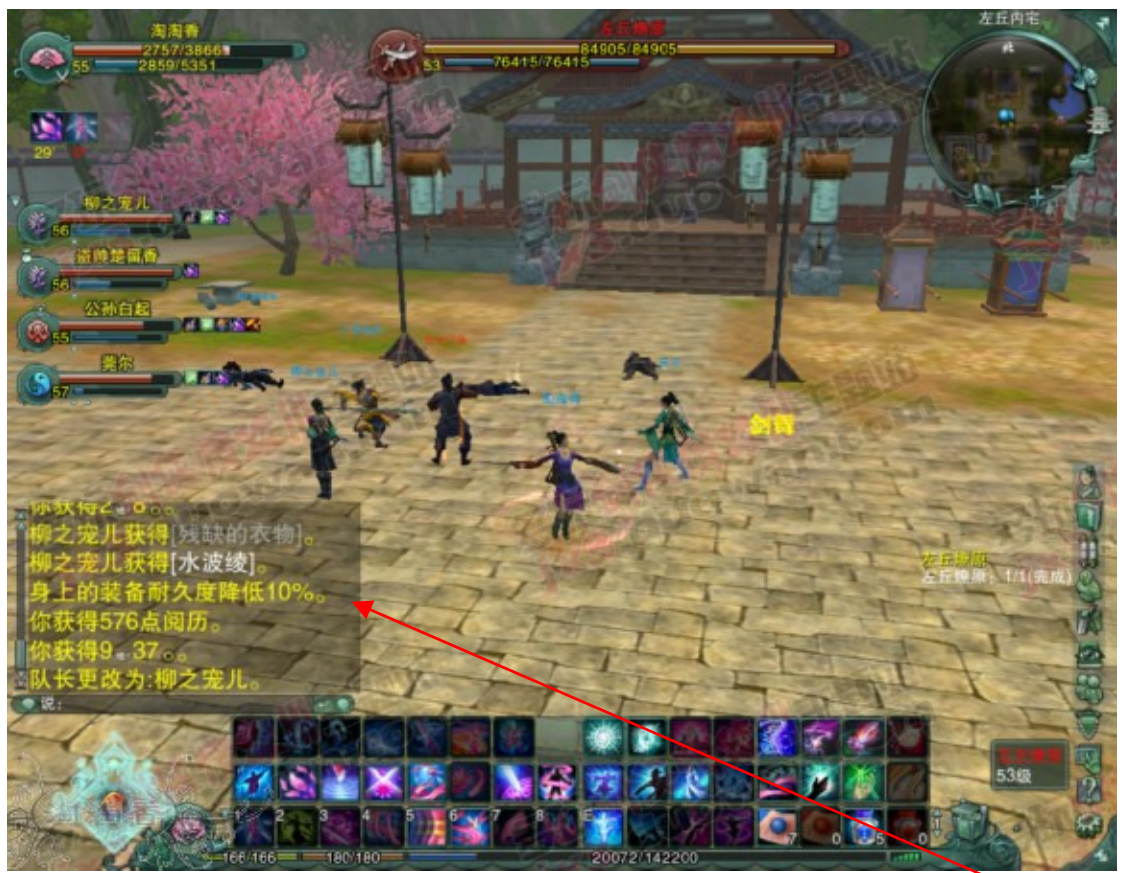

Figure 3: A mission in progress with dialogues with other players (permission obtained) 


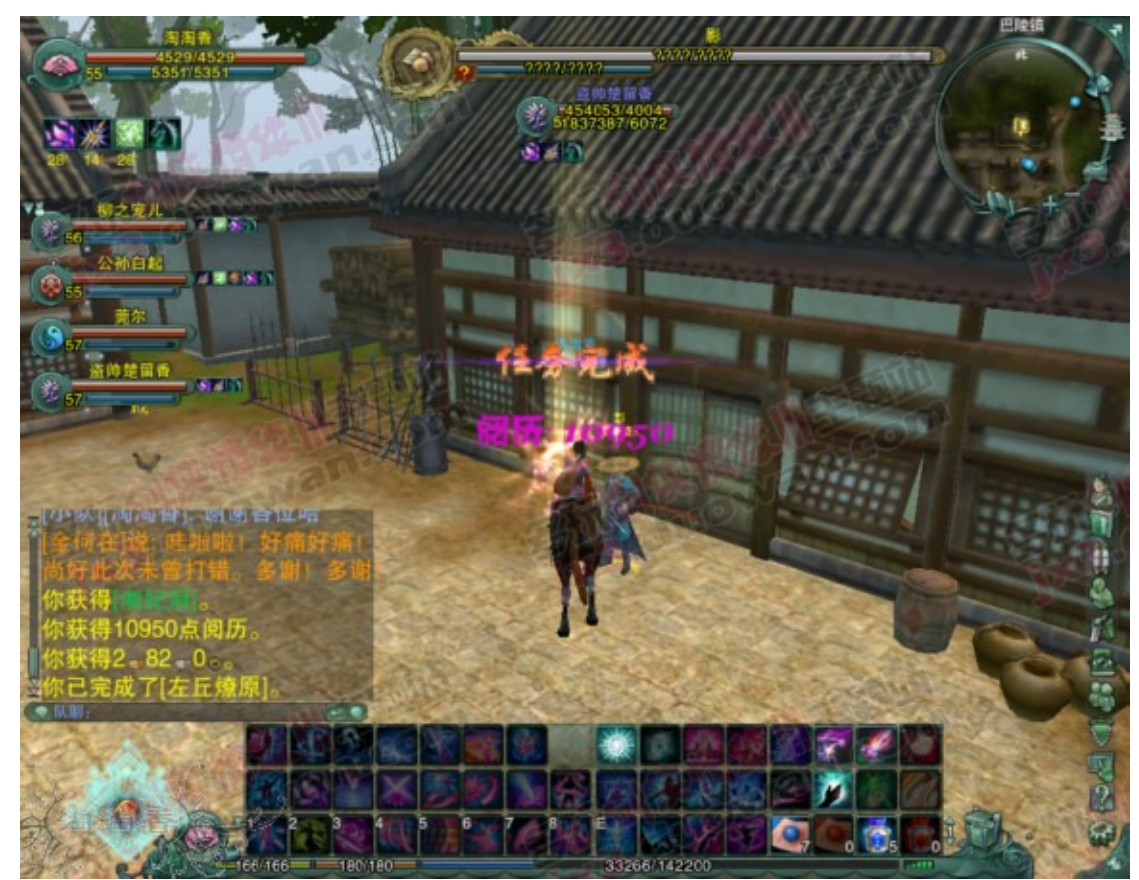

Figure 4: An example showing a mission is completed (permission obtained)

\subsection{Other games in demands}

There are other online VC games such as Warcraft and Diablo with similar goals and designs. Each member can play different characters, with one at a time. Each member can train up his/her skills, make friends and purchase tools or services to improve his/her abilities, lifespan and fighting experiences. However, there are very few in-depth literature in this study. Additional work is required to narrate the user experience of Warcraft (or Diablo) and make the comparisons with this chosen Cloud gaming VC. Member communities of these two games are growing. The sophisticated levels of technologies used in these games such as the use of Cloud computing can improve the response time, quality of service and the user experience (Rettberg, 2008; Barnett and Archambault, 2010; Chang and Wills, 2013). The success deliveries of these games can also support the case that gaming is an area for emerging Cloud.

\section{Related literature}

This section presents related literature to VCs including gaming communities and $\mathrm{C} 2 \mathrm{C}$ e-commerce websites.

\subsection{Definitions of virtual communities}

Fernback and Thompson (1995) recognized the importance of "social relationships" and defined VC as "social relationships forged in cyberspace through repeated contact within a specified boundary of space that is symbolically delineated by topic of interest". Hagel and Armstrong (1997) made distinctions between member-generated contents in 
VCs and content generated by service providers. Williams and Cothrel (2000) emphasized the common interests and goals as an important aspect of VCs.

Lee et al. (2003) define VC as "a virtual community is a cyberspace supported by computer-based information technology, centered upon communication and interaction of participants to generate member-driven contents, resulting in a relationship being built up." This is the closest to the definition in this paper. To sum up with Lee et al. (2003) and other research mentioned in the last paragraph, four common characteristics of a VC are observed:

- It exists in cyberspace

- It uses information technologies

- It is used for communication and interaction around common interests, and most of contents are user-generated

- It allows the formation of the social relationship.

There are four categories in VCs identified by Armstrong and Hagel (1996):

1. Interest communities in which people share an interest or an expertise on a specific topic to communicate with each other

2. Relationship communities in which people with similar experiences get together and establish meaningful relationships

3. Fantasy or gaming communities often refer to online games in which people come together to get fantastic or gaming experiences.

4. Transaction communities focus on transaction needs and where people can get trading information and advice. They can be classified as a business-to-business (B2B) or C2C communities.

\subsection{Trust in vendors/service providers and trust in virtual communities}

Trust has been recognized as a critical success factor in e-commerce presented by Lee and Turban (2001), Kim et al (2003) and Gefen and Straub (2004). Mayer et al (1995) define that trust is "the willingness of a party to be vulnerable to the actions of another party based on the expectation that the other will perform a particular action important to the trustor, irrespective of the ability to monitor or control that other party". Trust is multidimensional and ability, integrity and benevolence are the most cited three dimensions of trust (Gefen and Straub, 2004). Explanations are as follows. Ability is the competencies or the skills that make a trustee to be competent in a specific area. Integrity is the expectation that the trustee will act in accordance with social norms that he/she accepts. Benevolence is that the trustee will care and fulfill the trustor's request.

Two types of trust are identified in the study. Trust in the vendors/service providers and trust between members. Trust in the vendors means that the $\mathrm{C} 2 \mathrm{C}$ website (or VC sponsor) can provide quality services and can deliver consumers' demands upon payment. This is the assessment of institutional trust (and not individual trust), which can affect consumers' purchased behaviors (Gefen and Straub, 2004). Similarly, trust in ability, integrity and benevolence do apply. 
Trust between members can be a challenge to affect the prosperity and development of VCs, because many members can be anonymous and do not engage in face-to-face communications directly. Trust is thus a significant issue. Trust can be important in affecting members' behaviors since people can act more proactively when they trust the environment and people around it (Rothaermel and Sugiyama, 2001).

There are differences in different types of trust. Trust in online stores (Gefen, 2002), trust in the online vendor (Gefen et al., 2003 a; 2013 b) or trust in e-commerce (Lee and Turban, 2001) can be seen being different from trust in VCs that can be solely on interpersonal trusts (Ridings et al., 2002) - how much you trust the members and leaders in your VCs. It is a general trust towards others and the community and not to a specific person (although this happens, if there are terrible arguments online). People share their knowledge and experiences with other members they never met. This may mean a certain level of trust may exist because the information owner's privacy can be at risk, and the information seekers may be cheated by strangers. The model "antecedents-trustoutcomes" is used to investigate trust (Shankar et al, 2002). There are several classifications, where Gefen et al. (2003 a; 2013 b) classify the antecedents to trust into four types:

1. Knowledge-based trust, which focuses on trust-building through repeated interactions

2. Cognition-based trust, which focuses on trust building by the first impressions and reputation rather than interactions over a period of time.

3. Institution-based trust, which focuses on relying on an institution or third-party to build trust

4. Personality-based trust, which refers to individual personalities that influence trust building.

These four types are useful to adopt for different situations and cases. Another researcher has a different approach. Zucker (1986) identified three mechanisms to build trust: (1) process-based trust, which has similar definitions in knowledge-based trust; (2) characteristic-based trust, which implies that trust is established based on social similarities, such as ethnicities, families, religions or racial origins; and (3) institutionbased trust. Classifications from Gefen et al. (2003 a; 2013 b) and Zucker (1986) of trust antecedents are relevant to VCs although some revisions are required for adaptations to $\mathrm{VCs}$ and their trust building. For instance, members interacting with each other in computer-mediated communications can experience a trust building process similar to the one in the offline environment. When members get more familiar with each other, they can build up trust through interactions and resolving problems (such as killing big boss in the game; providing advice to other members for questions related to their online or offline activities). Similar interests among members can foster trust through activities. We combine two approaches to categorize the antecedents to trusts in VCs into knowledge-based, characteristic-based, institution-based and personality-based with details listed in Section 3. 
Ridings et al (2002) present a different case that the combined benevolence and integrity dimension can apply in trust in members, since both benevolence and integrity can maintain positive conversations in VCs. Trust in other members can encourage individual participations such as sharing knowledge and experience with others or receiving information from VC. Tung et al. (2001) discover that members with frequent involvements in VCs can be perceived as a greater representation of trust in the website or vendor than those less involved members. Subsequently, Smith (2002) explains that trust between members can create a positive influence that trust between members make others more willing to accept future recommendations from peer recommenders. In other words, the higher the trust between members, the higher the trust in the vendor or supplier with a positive word-of-mouth in the VC.

\section{Research model and hypotheses}

We present our hypotheses with their supporting literature and rationale. All hypotheses can be combined to represent different relationships in the different types of trusts, and factors that affect trust in VCs.

\subsection{Knowledge-based trust antecedent: familiarity}

Familiarity is the trust mechanism when members know each other more through interactions and activities and predict others' behaviors based on the information they obtain during their interactions. Familiarity can be used to describe the extent that consumers know about a website or a vendor, in which Gefen et al. (2003 a; 2013 b) explain the positive influence in the trust of the website and vendor. There is a positive relationship between interaction and trust. Rousseau et al (1998) classify several types of trust, and one type is the relational trust as a result of repeated interactions over time. Wallace (1999) found that frequent communications between team members can contribute to trust building, and the initial willingness to show trust can lead to the building of the actual trust. Ridings et al. (2002) point out the perceived responsiveness can positively relate to trust in other members in VCs. In summary of the literature to familiarity, members can get a high extent of accurate information and useful advice due to familiarity, which also reduces uncertainty and builds up trust in the long-term relationships. As a result, 2 related hypotheses are as follows.

H1a Familiarity with other members in a VC can positively influence trust in other members' 'integrity and benevolence'.

H1b Familiarity reduces uncertainty and builds up trust.

\subsection{Characteristic-based trust antecedent: perceived similarity}

Trust can be established based on common characteristics the trustor perceives of the trustee, which includes values, interests, religions and cultures. Perceived similarity offers the trust building mechanism on the common ground. Crosby et al. (1990) study an extensive literature in counseling, communications and psychology that relationship satisfaction is influenced by the similarity amongst individuals. Additionally, marketing 
researchers identify that shared beliefs and values about goals, policies and behaviors can affect trust significantly (Morgan and Hunt, 1994). Doney and Cannon (1997) illustrated that similarities between buyers and seller's salesperson can enhance trust and trading due to the common interests and values. The same relationship exists in the online environment. People are inclined to accept recommendations from members who are similar to them (Ziegler and Golbeck, 2007). In the grouped community with similar interests, people tend to perceive each other positively, which may enhance their trust beliefs (McKnight et al., 1998; 2002). Stewart (2003) demonstrates that perceived similarity between a trusted website and an unknown linked website has positive effects on trusting beliefs about the unknown target in his research on trust transfers on the Internet. His findings can show that trust can transfer through perceived similarity in different people.

Similar interests and goals can encourage trust building among members in VCs. Feng et al (2004) assert that online interpersonal trust can be influenced significantly by empathy accuracy, where the strength of empathy is related to the similarity the trustor identifies with the trustee. This paper focuses on the interests and values within the scope of perceived similarity as VCs are often established based on common interests and values, particularly Cloud-gaming VCs. Two hypotheses are as follows.

\section{H2a Perceived similarity with other members in a VC positively affect trust in other member's 'integrity and benevolence' \\ H2b Perceived similarity with other members in a VC build up trust}

\subsection{Institution-based trust antecedent: structural assurances}

Institution-based trust is a trust building mechanism for trusting and relying on a third-party organization involved in the process of trust and verification (Zucker, 1986). There are two types of trust, situation normality and structural assurance. Situation normality is a belief that the circumstance is normal and the transaction will be successful It means that people feel assured that everything is in a proper order (Baiser, 1986). VCs in China are highly similar in this context. In a research on online community users in China, the majority of 468 online communities used bulletin board systems (BBS), forums, instant messenger (IM) and blogs in the use of VCs. Additionally, 75.8\% of VC members had the university and college qualifications or above, and approximately $70 \%$ spent one to six hours per day in VCs (iResearch, 2006, 2007 a; 2012). In a market research conducted by Anastaya (2013), a consulting firm based in China and UK, results show that $56.2 \%$ of $\mathrm{VC}$ had the university and college qualifications or above, however, approximately $70 \%$ spent four to ten hours a day in VCs such as gaming VCs. Results show the movement in two trends. First, the use of the internet and VCs has reached out more people and it is not just a platform for "education divide" in China. Second, the same percentage of people have spent more time on VCs a day. Situation normality might not have much influence on members; trust, since more people have used the internet and have increased their number of hours per day to create more uncontrolled factors in the use of VCs. Situation normality is excluded in this study. 
Structural assurances refer to the beliefs that when contextual conditions such as regulations and guarantees exist in context, success is likely (McKnight et al., 1998; 2002). VCs have their own rules and regulations as a social network. For example, management of the community can have a positive impact on the member's engagement on transactions. In another example, leader's enthusiasm and involvement in community interactions can positively influence trust (Koh and Kim, 2004). In other words, excellent management in community rules, privacy guarantees and safety assurances provided by a VC can improve the extent of trust in members and VC providers. Members can have better confidence and trust in the website. Thus, hypotheses are as follows.

H3a. Perception of structural assurances positively affect trust in other members' 'integrity and benevolence'.

H3b. Perception of structural assurances positively affect trust in the website or vendor's integrity.

H3c. Perception of structural assurances can positively affect trust in the website or vendor's benevolence.

\subsection{Personality-based trust antecedent: trust propensity}

Trust propensity is known as disposition to trust, which reflects one's tendency to believe in others (Gefen et al., 2003 a; 2013 b). Ridings et al. (2002) state that trust propensity is "a general willingness based on extended socialization and life experience to depend on others". Mayer et al. (1995) assert that trust propensity's influence on trust, although it might be more effective when trustor is in the process of familiarizing with the trustee. To support this point, Lu and Zhou (2007) show that trust propensity can influence the initial trust in the website positively in a business-to-consumer (B2C) environment. Ridings et al. (2002) also identify that trust propensity has a positive relationship with trust in members in VCs. Thus, hypotheses are as follows.

H4a. Trust propensity positively affect trust in other members' 'integrity and benevolence'.

H4b. Trust propensity positively affect trust in the website/vendor's integrity.

H4b. Trust propensity can positively affect trust in the website/vendor's benevolence.

\subsection{Summary of hypotheses}

Four factors contributing to the trust were identified. Related literature in each factor has been presented, where hypotheses in each factor are identified. Rationale for contributing to the development of hypotheses has been explained. All these hypotheses will be validated through the analysis of the collected data presented in the next section.

\section{$5 \quad$ Methodology and results}

Descriptions in methodology are as follows. First, we demonstrate the use of VC to test our hypotheses. Second, we describe the process involved in the development of the 
research instrument. Third, we present the instrument validation and refinement process. In the last step, we show and explain the hypothesis testing results. The chosen community is a Cloud gaming VC.

\subsection{Survey questions}

Survey questions were designed and set up based on hypotheses presented in Section 4. This can ensure that relevant question for each hypothesis can be distributed to the target groups of audience, and results and comments received from the surveys could be used for analysis. This section aims to present the list of survey questions and the supporting rationale. The four hypotheses are classified under familiarity (FA), perceived similarity (PS), structural assurances (SA) and trust propensity (TP). Corresponding survey questions are presented in Table 1.

Table 1: The list of survey questions for four major hypotheses

\begin{tabular}{|c|c|c|}
\hline Items & Questions & Additional comments \\
\hline FA1 & $\begin{array}{l}\text { I become familiar with the IDs of some } \\
\text { members through reading posts, posting, or } \\
\text { replying to messages in the Cloud gaming } \\
\text { Community }\end{array}$ & $\begin{array}{l}\text { These four questions support } \\
\text { hypotheses presented in } \\
\text { Section 4.1. }\end{array}$ \\
\hline FA2 & $\begin{array}{l}\text { I become familiar with the interests and } \\
\text { behavioral characteristics of some members } \\
\text { such as their writing styles through reading, } \\
\text { posting, or replying to messages in the Cloud } \\
\text { gaming Community }\end{array}$ & $\begin{array}{l}\text { The preferred community is } \\
\mathrm{http}: / / \mathrm{j} \times 3 . x 0 y 0 . c o m \text {, and if not, } \\
\text { a similar role-based Cloud } \\
\text { gaming is required. }\end{array}$ \\
\hline FA3 & $\begin{array}{l}\text { I become familiar with other members through } \\
\text { reading, posting, or replying to messages in the } \\
\text { Cloud gaming Community }\end{array}$ & \\
\hline FA4 & $\begin{array}{l}\text { I communicated with some members in the } \\
\text { Cloud gaming Virtual Community frequently }\end{array}$ & \\
\hline PS1 & $\begin{array}{l}\text { I feel members in the Cloud gaming Virtual } \\
\text { Community have common goals }\end{array}$ & $\begin{array}{l}\text { These four questions support } \\
\text { hypotheses presented in } \\
\text { Section } 4.2 \text {. }\end{array}$ \\
\hline PS2 & $\begin{array}{l}\text { I feel members in the Cloud gaming Virtual } \\
\text { Community have similar interests to mine }\end{array}$ & \\
\hline PS3 & $\begin{array}{l}\text { I feel members in the Cloud gaming Virtual } \\
\text { Community have similar values to mine }\end{array}$ & \\
\hline PS4 & $\begin{array}{l}\text { I feel members in the Cloud gaming Virtual } \\
\text { Community have similar experience to mine }\end{array}$ & \\
\hline SA1 & $\begin{array}{l}\text { I am at ease communicating with other } \\
\text { members because Cloud gaming establishes } \\
\text { rules for the community }\end{array}$ & $\begin{array}{l}\text { These four questions support } \\
\text { hypotheses presented in } \\
\text { Section } 4.3 \text {. }\end{array}$ \\
\hline SA2 & $\begin{array}{l}\text { I am at ease communicating with other } \\
\text { members because there are administrators and } \\
\text { forum owners managing the Cloud gaming } \\
\text { Virtual Community }\end{array}$ & \\
\hline
\end{tabular}




\begin{tabular}{|l|l|l|}
\hline SA3 & $\begin{array}{l}\text { I feel safe communicating with other members } \\
\text { because Cloud gaming Virtual Community } \\
\text { provides Internet safety alerts }\end{array}$ & \\
\hline SA4 & $\begin{array}{l}\text { I feel safe communicating with other members } \\
\text { because I accessed Cloud gaming Virtual } \\
\text { Community through a well-known, reputable } \\
\text { portal }\end{array}$ & \multicolumn{1}{|l|}{} \\
\hline TP1 & I generally have faith in humanity & $\begin{array}{l}\text { These three questions support } \\
\text { Section 4.4. }\end{array}$ \\
\hline TP2 & I feel that people are generally reliable in \\
\hline TP3 & $\begin{array}{l}\text { I generally trust other people unless they give } \\
\text { me reason not to }\end{array}$ & \\
\hline
\end{tabular}

\subsection{Data collection}

Three methods were used to collect data from subjects who were members of the community. First, paper questionnaires were distributed to undergraduate and graduate students in a university in Chengdu, China. Second, an online version of the questionnaires was distributed for about four weeks to ensure sufficient data collection. Additionally, an allied to the VC community helped us distribute the online survey to their student members. All these three ways could ensure a sufficient sample size was collected. Out of approximately 800 people we sent out the questionnaires, altogether 120 responded the questionnaires, with $15 \%$ of return rate. Only 100 of them answered all the questions and provided useful feedback to our hypotheses, which means $83.33 \%$ of valid sample size.

Table 2 shows the descriptive information about the dataset. 55\% of the respondents were male and $45 \%$ were female. The majority (97\%) of the respondents were aged between 18 and 35 since the questionnaires were distributed to a university in Chengdu and student members in an allied VC. $98 \%$ of them had the education level as university undergraduate or above. Although well-educated young adults were surveyed in this study, they are the main shoppers of $\mathrm{C} 2 \mathrm{C}$ websites. Based on the research results, more than $60 \%$ of $\mathrm{C} 2 \mathrm{C}$ shoppers in China are between 18 and 30 years old. Similarly, more than $60 \%$ of Taobao shoppers have undergraduate education and above (iResearch, 2008). Although 100 is a small sample size comparing to the population of Chinese young adults, our data still shows a good representation of the market research result conducted by iResearch. In terms of membership history on VC, $20 \%$ of them were new members with member history of less than six months and $25 \%$ of them were senior members over three years. Although the sample had a high percentage of new members, $88 \%$ of them showed that they had purchased on Taobao. Taobao (2007) is a popular $\mathrm{C} 2 \mathrm{C}$ website like eBay that the most commonly purchased items include clothing and footwear, cosmetics and jewelry, and computers and accessories. All the respondents also required to rate their expected scores before the use of $\mathrm{VC}$ and the actual scores after the use of $\mathrm{VC}$, as well as the number of incidents and complaints that damaged their membership experience. 
In our study, the top three were computer and accessories, books and clothing and footwear if the participants use Taobao for general purposes and not for gaming. The reason that books were in the list was because the majority of respondents was students. Within computer and accessories, different items were listed. The top list is gaming equipment. It means that students who were on gaming VCs, they traded equipment for real money. They also traded their services, such as playing the game on behalf of the VC members for real money. This phenomenon has become a popular trend amongst students. Details will be presented in Section 5. The second in the list is the purchase of smart phone games. Although young adults still prefer free online games based on our survey results, some of respondents expressed that they also paid for games if they were excellent and their friends were on there. The third in the list is the computer peripheral equipment such as cables, protective covers for smart phones and iPad, USB, memory card, mouse and keyboard, which are important to students' daily life. Table 2 shows the sample demographics with six major categories: gender, age, education, the length of membership history, whether they have purchased online, types of products purchased and equipment purchased the most frequent.

Table 2: Sample demographics $(\mathrm{N}=100)$

\begin{tabular}{|llc|}
\hline Measure & Item & Count and percent \\
\hline Gender & Male & 62 \\
& Female & 38 \\
& 18 and below & 8 \\
& 19 to 24 & 55 \\
& 25 to 30 & 25 \\
Education & 31 to 35 & 10 \\
& 36 and above & 2 \\
& High school or below & 2 \\
Length of membership & Two-year college & 20 \\
history & Four-year university & 58 \\
& Graduate school and above & 20 \\
& 3 to 6 months 3 months & 8 \\
& 6 months to 1 year & 12 \\
Have purchased online & Yes & 15 \\
(such as Taobao) & 1 year to 2 years & 24 \\
Types of products & Oyears and 3 years & 16 \\
purchased (out of 88) & No & 25 \\
Equipment purchased & Tools for automated playing & 88 \\
the most frequent (out & & \\
\hline
\end{tabular}


of 84)

Tools for enhanced fighting $\quad 12$

Tools for enhanced life $\quad 30$

While more participants are males, the age distribution shows most of the participants are between 19 and 24, and then between 25 and 30, which correspond to their education level. The length of their membership history varies, and the majority are between 1 to 2 years, or over 3 years, which indicate that participants know this online gaming community fairly well. 88 out of 100 participants had purchased online. Amongst those who had $\mathrm{C} 2 \mathrm{C}$ e-commerce experience, equipment on games are the most popular items responded by 84 participants. Services on games means gamers paid someone to play on their behalf which had 4 counts. Amongst 84 participants, 42 said they purchased tools for automated playing the most frequent due to their busy university life, and they used those tools to ensure that their main characters can be in a continuous playing mode. The second choice was tools for the enhanced life, so that their played characters could have more supplies of blood and power and could preserve their lifeline longer.

\subsection{The data analysis of the collected data}

This section presents the data analysis of the collected data. Table 3 shows results for mean scores, standard deviations and p-values for all the items in the hypotheses. Explanations are as follows. The p-value should be below 0.05 to indicate that the results falls into the $95 \%$ of confidence interval.

Table 3: The mean scores and standard deviations (scores out of 5) for hypotheses

\begin{tabular}{|c|c|c|c|c|}
\hline Items & Mean & $\begin{array}{l}\text { Standard } \\
\text { deviations }\end{array}$ & $\begin{array}{l}\text { p-values (p } \\
\text { less than) }\end{array}$ & Remarks \\
\hline FA1 & 3.90 & 0.60 & 0.03 & $\begin{array}{l}\text { TP category has the higher standard } \\
\text { deviations in general. }\end{array}$ \\
\hline FA2 & 4.00 & 0.70 & 0.04 & \\
\hline FA3 & 3.70 & 0.60 & 0.03 & \\
\hline FA4 & 3.50 & 0.60 & 0.03 & \\
\hline PS1 & 4.20 & 0.40 & 0.02 & $\begin{array}{l}\text { PS category has a lower standard deviation in } \\
\text { general. }\end{array}$ \\
\hline PS2 & 4.40 & 0.30 & 0.02 & \\
\hline PS3 & 4.00 & 0.40 & 0.02 & \\
\hline PS4 & 3.90 & 0.50 & 0.03 & \\
\hline SA1 & 4.30 & 0.30 & 0.02 & $\begin{array}{l}\text { SA category has the lowest standard deviation } \\
\text { in general. }\end{array}$ \\
\hline SA2 & 4.40 & 0.30 & 0.02 & \\
\hline SA3 & 4.50 & 0.20 & 0.02 & \\
\hline SA4 & 4.40 & 0.30 & 0.02 & \\
\hline TP1 & 3.70 & 1.00 & 0.05 & $\begin{array}{l}\text { This has the widest standard deviations, since } \\
\text { some members had an unpleasant experience } \\
\text { and some had the opposite. }\end{array}$ \\
\hline TP2 & 3.80 & 0.80 & 0.05 & TP category has the highest standard \\
\hline
\end{tabular}




\begin{tabular}{|l|l|l|l|l|}
\hline & & & & deviations. \\
\hline TP3 & 4.00 & 0.50 & 0.05 & \\
\hline
\end{tabular}

In the category for the first hypotheses (FA1 to FA4), the mean values are between 3.50 and 3.90 with a standard deviation (SD) between 0.60 and 0.70 , which is an acceptable range. The mean scores in FA1 and FA4 are the lowest amongst all the other categories. This may mean that among 100 valid samples, some participants have varied opinions. The p-values between FA1 and FA4 are low and below 0.05, which means the probability of the null hypotheses (that against the hypotheses) is low. In the category for the second hypothesis (PS1 to PS4), the mean scores are between 3.90 and 4.20 with a smaller range of SD between 0.30 and 0.50 . All p-values are low and below 0.05.

In the category for the third hypotheses (SA1 to SA4), the mean scores are between 4.30 and 4.50 with a smaller range of SD between 0.20 and 0.30 . This category has the most positive outcomes with the least of SD in this group of 100 participants. The pvalues have the lowest values in all four categories to further support this observation. A likely explanation is that when members establish trust with other members, they find that the ease and the flow of communications are mutual, interactive and effective as indicated by some comments left in the survey. They talk about games and strategies but also their personal life. In the category for the fourth hypotheses (TP1 to TP3), the mean values are between 3.70 and 4.00 with the large range of SD between 0.50 and 1.00. One likely explanation is that some participants fully agree with fourth hypothesis and have rated 5 out of 5 . In contrast, some participants take a more conservative approach and rate scores between 3 and 4 . In other words, participants have split views about the extent of the positive impacts, although the majority has the positive views about the fourth hypothesis. All these results show that the participants acknowledge the positive values of the fourth hypothesis with varying extents.

\subsection{Data analysis while comparing the current and previous collected data}

Table 4 presents the key inputs for comparison. The lead author had a previous consultancy involved with this research, except with a different group of participants. Due to the agreement, details of the work are not enclosed in this paper. However, the mean scores of the previous data can be used as a baseline, which means the target scores for each category in the survey. 
Table 4: Comparison of the current and previous data (scores out of 5)

\begin{tabular}{|l|l|l|l|l|}
\hline Items & $\begin{array}{l}\text { Mean scores of the } \\
\text { current data (mean } \\
\text { from the survey) }\end{array}$ & $\begin{array}{l}\text { p-values (p } \\
\text { less than) of } \\
\text { the current } \\
\text { data }\end{array}$ & $\begin{array}{l}\text { Mean scores of the } \\
\text { previous data (from the } \\
\text { previous consultancy) }\end{array}$ & $\begin{array}{l}\text {-values (p } \\
\text { less than) of } \\
\text { the previous } \\
\text { data }\end{array}$ \\
\hline FA1 & 3.90 & 0.03 & 3.80 & 0.03 \\
\hline FA2 & 4.00 & 0.04 & 3.80 & 0.05 \\
\hline FA3 & 3.70 & 0.03 & 3.80 & 0.04 \\
\hline FA4 & 3.50 & 0.03 & 3.60 & 0.03 \\
\hline PS1 & 4.20 & 0.02 & 4.10 & 0.02 \\
\hline PS2 & 4.40 & 0.02 & 4.30 & 0.02 \\
\hline PS3 & 4.00 & 0.02 & 4.00 & 0.02 \\
\hline PS4 & 3.90 & 0.03 & 3.80 & 0.03 \\
\hline SA1 & 4.30 & 0.02 & 4.10 & 0.01 \\
\hline SA2 & 4.40 & 0.02 & 4.20 & 0.01 \\
\hline SA3 & 4.50 & 0.02 & 4.20 & 0.01 \\
\hline SA4 & 4.40 & 0.02 & 4.00 & 0.02 \\
\hline TP1 & 3.70 & 0.05 & 3.60 & 0.05 \\
\hline TP2 & 3.80 & 0.05 & 3.70 & 0.05 \\
\hline TP3 & 4.00 & 0.05 & 4.00 & 0.06 \\
\hline Mean & 4.05 & 0.03 & 3.93 & 0.03 \\
\hline
\end{tabular}

Baseline from the previous record has been widely adopted. One example is the stock market exchange that the baseline is based on the previous day's trading index values. The $\mathrm{p}$-values for the current and previous data are included in Table 4. Although the mean of $\mathrm{p}$-values for both current and previous data are below 0.03, the individual $\mathrm{p}$ values in each item is different. The overall trend is similar in a way that the category between SA1 and SA 4 has the lowest p-values and the category between TP1 and TP3 has highest $\mathrm{p}$-values. In terms of the p-values for the current data, they are 0.05 and below. This means that results supporting hypotheses fall within $95 \%$ confidence interval the the probability of the null hypothese are below $5 \%$. Similarly, results of the previous data fall within $95 \%$ confidence interval, except value in TP3. The overall trend is that results support the hypotheses due to the low p-values presented. However, additional test or analysis is required to further support this. See Section 6.1 for details.

\section{Discussion}

This section presents topics of discussion including the interpretation of the analysis and the limitations of this research.

\subsection{Advanced statistical analysis: One-Way ANOVA}

This section describes the statistical analysis performed by Analysis of Variance (ANOVA), which is a useful method to analyze two sets of data, particularly if these two sets of data involved with two different groups (Chang and Wills, 2013). The data presented in Table 4 meets this criteria, since it includes current data with this group of 
cohorts and the previous data with another group of cohorts. The One-Way ANOVA is chosen because it was the same survey for two different groups of gamers who do not have huge difference in the culture and the language of communication on the Cloug gaming. Statistical results are computed by STATA 11, which is an statistical package used by researchers, statisticians and industry. The variable "previousdata" is referred to the mean scores of the previous data and the variable "currentdata" is referred as the mean scores of the current data. One-way ANOVA is used to compare the results based on the points of the view offered by "currentdata". The term SS is referred to sums of squared errors, $\mathrm{df}$ is referred to degree of freedom, MS is referred as the mean squared errors and $\mathrm{F}$ is the result of the F-test. In terms of accuracy measurement, values in SS and MS should be as low as possible to indicate that all the sums of errors are low in values. F-test should have a higher value. This is reflected in the term Prob $>$ F, which corresponds to the p-value of this data analysis (Hull, 2009; Lee et al., 2010). The p-value should be below 0.05 to indicate that the results falls into the $95 \%$ of confidence interval. The low p-value suggests that the probability of getting the null hypothese is low. Rsquared value is to indicate how fit the regression line is. It should be above 0.5 and being as close as to 1 to show that results are consistent and do not have large discrepancies (Lee et al., 2010). The value of R-squared is 0.8602 to show that result of regression is excellent. Intraclass correlation shows the correlation relationship between the currentdata and previousdata and it should be above 0.5 to show that there are close correlation between two data. The estimated stadandard deviations (SD) for previousdata and currentdata is 0.268 and 0.152 respectively. Both SDs are low to support that the difference between all mean scores are low and fairly consistent. All these results are very positive to support to our four hypotheses.

Table 5: Results of the analysis

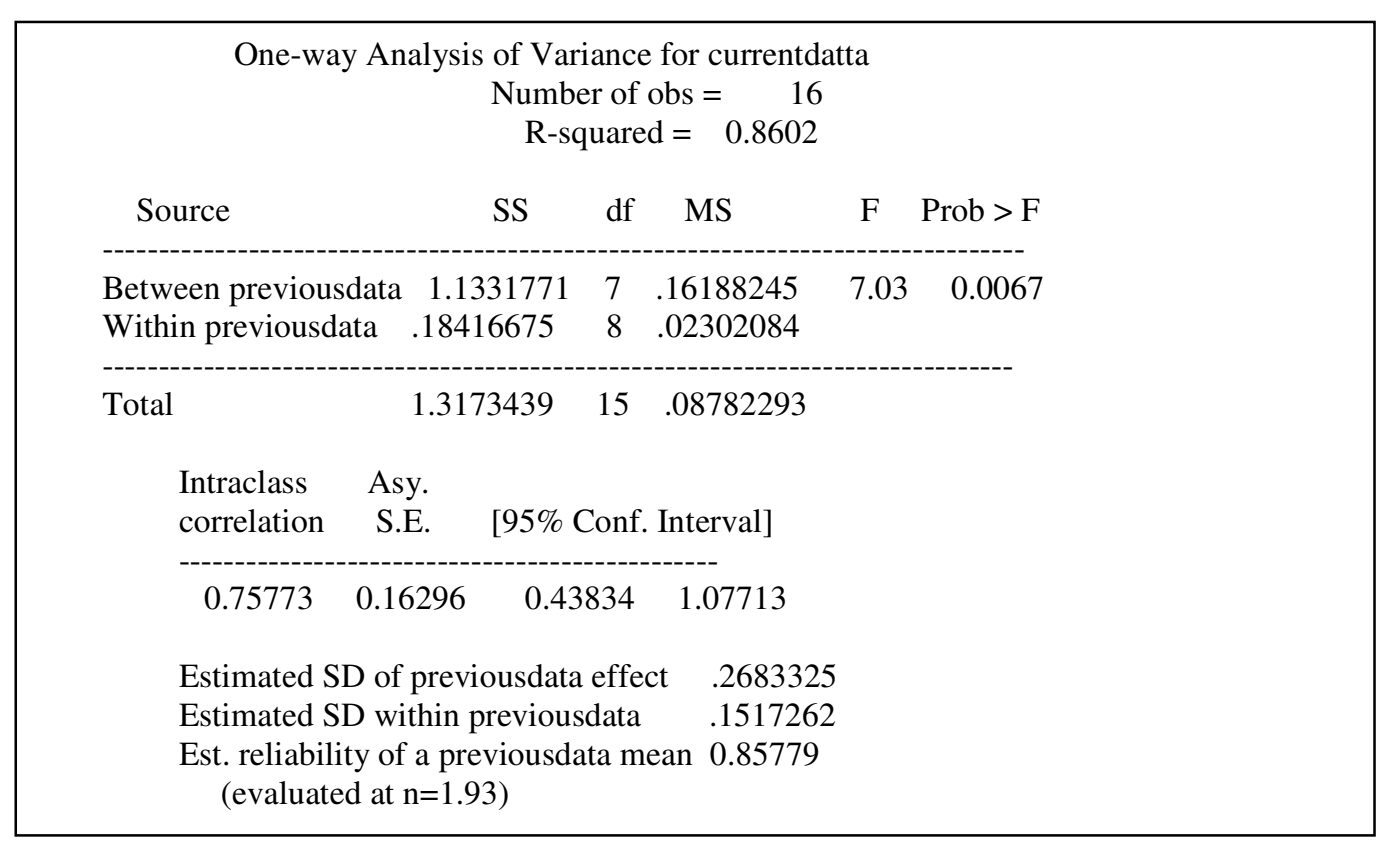




\subsection{Gaming is an emerging aspect of Cloud Computing}

As discussed in Section 1, online gaming has a huge market potential and development with rapid increases in revenues and user community year by year. To support millions of users with the real-time support, Cloud Computing has become a popular option to offer multi-tenancy and dynamic scaling of servers with virtual machines. When a virtual machine is in demand, the Cloud can check which server is free of virtual machine and dynamically assign the virtual machines to the players, so that when the resource can be flexibly assigned to ensure that the rate of utilization can be high. This approach is called the resource allocation model which identifies which part of the Cloud needs more demands, and then assigns players to places with less demands.

Although the increase in membership numbers is seen in the online community, trust is a main concern among some participants. Our study can illustrate the significance in this area. While trust is a factor which can be different based on the communities, the culture and the mission of the communities, our work presents the case that online gaming is an aspect of emerging Cloud. In this section, we aim to illustrate why online gaming can attract much attention and has created a platform to develop friendship and business opportunities. Instead of surveying another group of 100 participants with probable different outcomes, we use a walkthrough approach to explain why online gaming has the potential and the market for Cloud Computing.

\subsection{Limitations of this research}

Limitations of this research are as follows. First, it only collects 100 valid survey results and a greater sample size is preferred, so that results can represent opinions from a larger group of audience. Second, this game is created in China and all the players are Chinese players mainly in mainland China (about 99\%). It is only a relevant case study reflecting the gaming VC in China. Third, the data collection took place in Chengdu, a city in South-West China. Even if this study is focused on the Chinese analysis on gaming, surveys in more cities should be undertaken in the forthcoming research.

\section{$7 \quad$ Future Directions of this research}

This section presents future directions of this research to address all the limitations imposed by the current research method.

\subsection{Transforming trust to friendship and business}

This section aims to strengthen the benefits of achieving high extent of trust in the gaming VCs, which includes friendship and business opportunities. In the process of the data collection for this paper, 20 participants provided comments in the opinion boxes that they could make friends through this game and some of them not only met each other but also had their social activities together. They said that they had developed a strong friendship since they had goals and interests in common. Additional five short interviews were taken and the results had the identical feedback. These five participants could 
provide details about how the gaming VC had changed their life in positive ways and they had good times with friends they met on this gaming website.

The next benefit is the rising business opportunities from the gaming VCs. About 10 participants left the comment boxes that they could sell tools and their services, such as helping other players to train up their skills. Participants declined to be interviewed, although two of them claimed to earn sufficient financial support through their gaming consultancy services but both did not want to reveal further information. While more data and information should be collected through more interviews, the next stage of this research is to identify two groups of players particularly the second type including:

1. Players who have developed a strong friendship in the gaming VCs

2. Players who have developed successful business models in the gaming VCs

\subsection{Inclusion of another Cloud-based gaming community}

A useful alternative is to include another Cloud-based gaming community to identify any similarities and differences between two Cloud-based game communities which include different ethnic groups and cultures of the VCs. The chosen gaming VC is based in China and even more groups of participants are used, it is only reflecting the case studies of Chinese Cloud gaming VC. A western game such as Warcraft is planned to be the case study, since it involves players from different countries. The case study of trust building is highly relevant in a way that can involve with people living in different countries, time zone and culture differences. In this way, differences caused by the culture, different groups of participants and games can be minimized. Since trust building can be a time-consuming process, research methods such as interviews and case studies should be used to help the lead researcher know more about the players in person and understand how they work together as a successful team, as well as how players develop trust building through the activities they have undertaken in both online and offline environments. In this way, aims to investigate the two groups of players identified in Section 7.1 can be achieved.

\subsection{Risk towards trust-building}

According to Sharpe (1990), there are two types of risks: uncontrolled and controlled risks in all the projects involved. Trust building is exposed to the influence of risks, particularly to what extent that a managed risk can become uncontrolled, and the investigations about how to calculate the uncontrolled risk. All these concerns have to be taken into considerations in dealing with trust building. Trust between people are involved with risk, and thus future development in this area is required. Chang (2014 a) also explains the use of Business Intelligence as a Service to calculate both uncontrolled and controlled risks in the market, which are essential to the market and information security management. Related discussions bout using a model for risk analysis are in the next section. 


\subsection{Deploy another model for data analysis}

The current data analysis relies on a tool to get all the statistical results. A good model should be used, so that it can help researchers identify key inputs to be measured and provide more comprehensive interpretations of the outputs. There are a number of models to be studied. Our previous work includes the use of Organizational Sustainability Modeling (OSM), a model developed by Chang (2013) based on the improvement of Capital Asset Pricing Model (CAPM). CAPM neglects the computational competency to analyze the large datasets and also does not address the measurement of risk for Computing or organizations that adopt new technology. The researcher (Chang, 2013) published a number of papers together with his colleagues before obtaining his doctorate (Chang et al., 2011 a; 2011 b). To consolidate this work, several case studies have been presented. The aim is to show that OSM can be used to analyze how organizations adopt Cloud with the investigations about their status of return (improvement in efficiency; profitability; improvement in user satisfaction) and risk (uncontrolled risk and managed risk) (Chang et al., $2011 \mathrm{a} ; 2011 \mathrm{~b}$ ). Additional effort has been spent on the development of OSM for mobile customer satisfaction measurement (Chang, 2014 b; 2014 c), in which the results have been effective for the stakeholders and potential investors to understand the status of return and risk associated with mobile Cloud services.

Organizational Sustainability Modeling (OSM) will be used to analyze the status of return and risk for the future research as follows. First, OSM can calculate the uncontrolled risk, beta, which can largely influence the trust building in progress. Current literature does not address about the uncontrolled risk for trust building. There is a need for a chosen model such as OSM to define what to measure, provide a guideline about the computational process and offer techniques of performing data analysis. Second, OSM also provides a recommendation about the management the controlled risk which is essential to trust and trust building.

\section{Conclusion}

Trust is an important issue in the virtual communities (VCs), particularly when there are incidents of frauds, cheats and selling inferior-quality products among members and malicious closing-down of service providers. With the rise of Cloud gaming VCs, purchasing items or services become popular options for players for the improvement of abilities, experiences and lifespan. To further understand this topic, we studied a number of literature. We identified that there were four major unexploited areas in trust, and we therefore carefully set up our four major hypotheses. The survey was the research method and questions were designed based on our hypotheses. We sent out our surveys to university students in Chengdu, China, and collected 100 valid sample size. We presented the mean scores for each category of the four hypotheses and explained the demographics, as well as the likely reasons for large standard deviations and mean scores in some categories of the hypotheses. We presented the results and explained their interpretations. W used one-way ANOVA to compare the current and previous data, as well as their explanations. Results show that there is a high extent of accuracy reflected by the low standard deviations, low mean squared errors and low sum of squared errors. 
High R-squared values show that all the data fit to the regression line in the analysis. The low p-value suggests that the probability of getting the null hypothesis is low. Results also support our four hypotheses.

Cloud gaming VCs have become more popular with the rapid rise in the user communities. Trust still remains a main concern in those VCs. To support the case that online gaming is an aspect of emerging Cloud, we provided a walkthrough about how to play the game, how to interact with other members, how to purchase the tools from other members and how such actions could benefit the mission to be completed. Topics such as the role-playing game, mission in the game, transferring trust to friendship and other games in demands have been covered and explained. We also describe the limitations of our research since it is currently focused on China and is on the study of one game. We plan to investigate another game, Warcraft, and study any similarities and differences between two Cloud-based games. We will study the impact of risk towards trust building and use models to analyze their effects. Additionally, some participants left their detailed opinions on comments boxes but declined to be interviewed. We plan to understand how players who have developed a strong friendship in gaming VCs, as well as players who have developed successful business models in gaming VCs in our future research.

\section{References}

Analysys. 2008 survey report on online users of retail market in China. 2008.

Anastaya, report on gaming virtual communities, white paper, 2013.

Armstrong, A., and Hagel, J. The real value of on-line communities. Harvard Business Review, 74, 3, 1996, 134-141.

$\mathrm{Ba}, \mathrm{S}$. Establishing online trust through a community responsibility system. Decision Support Systems, 31, 3, 2001, 323-336.

Barnett, J. H., \& Archambault, L. How massive multiplayer online games incorporate principles of economics. TechTrends, 54(6), 29, 2010.

Bughin, J., and Zeisser, M. The marketing scale effectiveness of virtual communities. Electronic Markets, 11, 4, 2001, 258-262.

Chang, V., De Roure, D., Wills, G., \& Walters, R. Case studies and organisational sustainability modelling presented by cloud computing business framework. International Journal of Web Services Research, 8(3), 2011 a, 26-53.

Chang, V., De Roure, D., Wills, G., John Walters, R., \& Barry, T. Organisational Sustainability Modelling for Return on Investment (ROI): Case Studies presented by a National Health Service (NHS) Trust UK. CIT. Journal of Computing and Information Technology, 19(3), 2011 b, 177-192. 
Chang, V., Wills, G., Walters, R., \& Currie, W. Towards a structured Cloud ROI: The University of Southampton cost-saving and user satisfaction case studies, In, Sustainable ICTs and Management Systems for Green Computing., IGI Global, 2012, 179-200.

Chang, V. A proposed model to analyse risk and return for a large computing system adoption, 2013, Doctoral dissertation, University of Southampton.

Chang, V., Walters, R. J., \& Wills, G. The development that leads to the Cloud Computing Business Framework. International Journal of Information Management, 2013, 33(3), 524-538.

Chang, V., \& Wills, G. A University of Greenwich Case Study of Cloud Computing. ELogistics and E-Supply Chain Management: Applications for Evolving Business, 2013, 232.

Chang, V., The Business Intelligence As a Service in the Cloud. Future Generation Computer Systems, 37, 512-534, 2014 a.

Chang, V., The Big Data Analysis for measuring popularity in the Mobile Cloud. In, The first international workshop on Emerging Software as a Service and Analytics, ESaaSA 2014, Barcelona, Spain, 3 - 5 Apr, 2014 b.

Chang, V. Measuring and analyzing German and Spanish customer satisfaction of using the iPhone 4S Mobile Cloud service. Open Journal of Cloud Computing, 1(1), 19-26, 2014 c.

Crosby, L. A., Evans, K. R., and Cowles, D. Relationship quality in services selling: an interpersonal influence perspective. Journal of Marketing, 54, 3, 1990, 68-81.

Doney, P. M., and Cannon, J. P. An examination of the nature of trust in buyer-seller relationships. Journal of Marketing, 61, 2, 1997, 35-51.

Feng, J., Lazar, J., and Preece, J. Empathic and predictable communication influences online interpersonal trust. Behavior and Information Technology, 23, 2, 2004, 97106.

Fernback, J., and Thompson, B. Virtual communities: Abort, retry, failure? 1995. Available at http://www.well.com/user/hlr/texts/VCcivil.html, accessed on 4 July, 2014.

Fitzpatrick, M. China: Fake goods vendors switch to online selling. 2006. Available at http://www.just-style.com/article.aspx?id=93248, accessed on 4 July, 2014.

Fotis, J., Buhalis, D., \& Rossides, N. (2011). Social media impact on holiday travel planning: The case of the Russian and the FSU markets. International Journal of Online Marketing (IJOM), 1(4), 1-19. 
Gefen, D. E-commerce: the role of familiarity and trust. Omega: The International Journal of Management Science, 28, 6, 2000, 725-737.

Gefen, D. Reflections on the dimensions of trust and trustworthiness among online consumers. ACM SIGMIS Database, 33, 3, 2002, 38-53.

Gefen, D., Karahanna, E., and Straub, D. W. Inexperience and experience with online stores: the importance of TAM and trust. IEEE Transactions on Engineering Management, 50, 3, 2003a, 307-321.

Gefen, D., Karahanna, E., and Straub, D. W. Trust and TAM in online shopping: an integrated model. MIS Quarterly, 27, 1, 2003b, 51-90.

Gefen, D., and Straub, D. W. Consumer trust in B2C e-commerce and the importance of social presence: experiments in E-products and E-services. Omega: The International Journal of Management Science, 32, 6, 2004, 407-424.

Hagel, J., and Armstrong, A. Net Gain: Expanding Market through Virtual Communities. Harvard Business School Press, Boston, 1997.

Hull, J. Options, futures and other derivatives. Pearson education. 2009.

iResearch. Internet community user research report. 2006.

iResearch. The age structure of C2C shoppers in China. 2007a.

iResearch. China online social network research report. 2007b.

iResearch. China social network developing research report. 2008.

iResearch. China online social network research report. 2012.

Kim, D. J., Song, Y. I., Braynov, S. B., and Rao, H. R. A multidimensional trust formation model in B2C e-commerce: A conceptual framework and content analyses of academia/practitioner perspectives. Decision Support Systems, 40, 2 , 2003, 143-165.

Koh, J., and Kim, Y. G. Sense of virtual community: a conceptual framework and empirical validation. International Journal of Electronic Commerce, 8, 2, 2004, 7593.

Lee, C.F., Lee, A.C. and Lee, J. (Ed.), Handbook of Quantitative Finance and Risk Management, 1, Springer publisher, ISBN 978-0-387-77116-8, (2010).

Lee, F. S. L., Vogel, D., and Limayem, M. Virtual community informatics: a review and research agenda. Journal of Information Technology Theory and Application 
(JITTA), 5, 1, 2003, 47-61.

Lee, M. K. O., and Turban, E. A trust model for consumer internet shopping. International Journal of Electronic Commerce, 6, 1, 2001, 75-91.

Lehdonvirta, V. (2009). Virtual item sales as a revenue model: identifying attributes that drive purchase decisions. Electronic Commerce Research, 9(1-2), 97-113.

Lin, H. F. Understanding behavioral intention to participate in virtual communities. CyberPsychology \& Behavior, 9, 5, 2006, 540-547.

Lu, Y., and Zhou, T. A research of consumer's initial trust in online stores in China. Journal of Research and Practice in Information Technology, 39, 3, 2007, 167-180.

Mayer, R. C., Davis, J. H., and Schoorman, F. D. An integrative model of organizational trust. Academy of Management Review, 20, 3, 1995, 709-734.

McKnight, D. H., Choudhury, V., and Kacmar, C. The impact of initial consumer trust on intentions to transact with a web site: a trust building model. Journal of Strategic Information Systems, 11, 3-4, 2002, 297-323.

McKnight, D. H., Cummings, L. L., and Chervany, N. L. Initial trust formation in new organizational relationships. Academy of Management Review, 23, 3, 1998, 473490.

Morgan, R., and Hunt, S. D. The commitment-trust theory of relationship marketing. Journal of Marketing, 58, 3, 1994, 20-38.

Rettberg, J. W. (2008). Quests in World of Warcraft: Deferral and repetition. Digital culture, play, and identity: A World of Warcraft reader, 167-184.

Ridings, C. M., Gefen, D., and Arinze, B. Some antecedents and effects of trust in virtual communities. Journal of Strategic Information Systems, 11, 3-4, 2002, 271-295.

Rothaermel, F. T., and Sugiyama, S. Virtual internet communities and commercial success: individual and community-level theory grounded in the atypical case of TimeZone.Com. Journal of Management, 27, 3, 2001, 297-312.

Rousseau, D. M., Sitkin, S. B., Burt, R. S., and Camerer, C. Not so different after all: a cross-discipline view of trust. Academy of Management Review, 23, 3, 1998, $393-$ 404.

Shankar, V., Urban, G. L., and Sultan, F. Online trust: a stakeholder perspective, concepts, implications, and future directions. Journal of Strategic Information 
Systems, 11, 3-4, 2002, 325-344.

Sina, T. Sina survey reveals that more than $70 \%$ of the web users had ever bought fake products online. 2009. Available at http://www.kuqin.com/shuoit/ 20090313/39603.html.

Smith, D. N. Trust Me, Would I Steer You Wrong? The Influence of Peer Recommendations within Virtual Communities. Doctoral Thesis, University of Illinois, Chicago, 2002.

Stewart, K. J. Trust transfer on the World Wide Web. Organization Science, 14, 1, $2003,5-17$.

Taobao.com. A brief introduction to Taobao. Available at http://www.taobao. com/about/intro.php , accessed on July 4, 2014.

Tung, L. L., Tan, P. L. J., Chia, P. J. T., Koh, Y. L., and Yeo, H. L. An empirical investigation of virtual communities and trust. In Twenty-Second International Conference on Information Systems, 2001.

Wallace, P. The Psychology of the Internet. Cambridge University Press, Cambridge, UK, 1999.

Wang, H. Fake goods dealers find haven on internet, article, 2009. Available at http:// www.chinadaily.net/bizchina/2009-02/10/content_7459200.htm, , accessed on 4 July, 2014.

Williams, R. L., and Cothrel, J. Four smart ways to run online communities. Sloan Management Review, 41, 4, 2000, 81-91.

Ziegler, C. N., and Golbeck, J. Investigating interactions of trust and interest similarity. Decision Support Systems, 43, 2, 2007, 460-475.

Zucker, L. G. Production of trust: Institutional sources of economic structure. In B. M. Staw and L. L. Cummings (eds.), Research in Organizational Behavior, JAI Press, Greenwich, CT, 1986, 53-111. 